\title{
The relation between bacterial degradation of viscous polysaccharides and stool output in human beings
}

\author{
BY J. TOMLIN AND N.W. READ* \\ Sub-Department of Human Gastrointestinal Physiology and Nutrition, \\ Royal Hallamshire Hospital, Sheffield S10 $2 \mathrm{JF}$
}

(Received 14 April 1988 - Accepted 27 July 1988)

\begin{abstract}
1. The relation between bacterial degradation of three viscous polysaccharides (guar gum, ispaghula and xanthan gum) by colonic bacteria in vitro and their effects on colonic function were investigated by comparing the results of anaerobic in vitro incubations with fresh faeces from seven healthy volunteers (measuring viscosity, $\mathrm{pH}$ and gas production) with the effects of feeding all three polysaccharides to the same volunteers for 1 week each $(14-15 \mathrm{~g} / \mathrm{d})$ on faecal mass and whole-gut transit time.

2. Guar gum was rapidly fermented in vitro by faecal bacteria from all volunteers with concomitant loss of viscosity, reduction in $\mathrm{pH}$ and generation of gases. Ispaghula maintained its viscosity during incubation, but the $\mathrm{pH}$ fell significantly. The results of xanthan gum incubations showed considerable individual variation.

3. Only ispaghula significantly increased faecal mass, whilst none of the gums significantly affected stool frequency or transit time. Statistical analysis of the pooled results showed that although transit time and faecal output were inversely related, feeding viscous polysaccharides could influence these indices independently. Stool frequency was significantly correlated with the transit time, but not the faecal output.

4. Transit time was reduced by gum feeding to a significantly greater extent in those subjects whose faecal bacteria reduced or removed the viscosity of that gum, than in those subjects where the viscosity was maintained. In contrast, there was a smaller increase in faecal mass when the viscosity of the appropriate cultures was removed than when it was maintained or reduced. Increases in stool frequency were significantly associated with hydrogen production from in vitro cultures.
\end{abstract}

Ingestion of some undigestible viscous food polysaccharides produces larger and softer stools and reduces transit time, whilst other polysaccharides have little effect on these characteristics. There appear to be no consistent correlations between the chemical composition and physical structure of the polysaccharides and their physiological effects (Eastwood et al. 1986). In addition, the same polysaccharide can have disparate effects in different individuals. This suggests that these variations may be explained by differences in the breakdown of these polysaccharides by each individual's unique colonic bacterial flora. A study which introduced ispaghula husk into the diets of four volunteers found a very strong correlation in three of the volunteers $(r-0.999, P<0.05)$ between the extent of ispaghula digestion and the increase in faecal mass (Prynne \& Southgate, 1979). In vitro incubation studies have shown that as well as being degraded by the bacteria to different extents, fermentation of different polysaccharides produces distinct patterns of shortchain fatty acids and gases (Tomlin, 1987).

To investigate whether the degradation of viscous polysaccharides by colonic bacteria determines their effects on colonic function, three purified polysaccharides, guar gum, xanthan gum and ispaghula, were given to healthy volunteers for periods of 1 week. Faecal mass and whole-gut transit times were compared with the results of in vitro incubations of the polysaccharides with faecal samples collected from each of the volunteers before feeding the polysaccharide. 
Table 1. Dietary instructions given to volunteeers

Foods to exclude:

Bran and bran products

Wholemeal flour, bread, pasta, etc.

Wholegrain rice

Any food known to cause flatulence or a noticeable alteration in bowel habit, or both (e.g. hot curry)

Foods to restrict:

Beans; e.g. butter (Phaseolus lunatus), haricot (Phaseolus vulgaris), red kidney (Phaseolus vulgaris),

baked beans in tomato sauce

Chickpeas (Cicer arietinum), lentils (Lens esculenta), etc.

Nuts (one small bag; $50 \mathrm{~g} / \mathrm{d}$ )

Peanut butter (enough for one slice of bread/d)

Crisps (one small bag; $28 \mathrm{~g} / \mathrm{d}$ )

Weetabix, Shredded wheat, Cornflakes, etc (one helping/d)

Alcohol (no more than 4 units/d, no more than 12 units/week)*

* One alcohol unit is equivalent to $0.5 \mathrm{pt}(284 \mathrm{ml})$ beer, lager, cider, etc.; one glass $(142 \mathrm{ml})$ wine; one measure (43 g) spirits.

\section{METHODS \\ Experimental design}

Studies were carried out in six male and one female healthy volunteers, who ate a normal mixed diet and had not taken antibiotics during the previous 6 months. They gave written informed consent to the study, which was approved by the local Ethical Committee (Sheffield Area, Trent Regional Health Authority). The study in the male volunteers lasted 6 weeks in total. The 1 st week provided basal values. The 2 nd, 4 th and 6 th weeks were the test weeks during which the viscous polysaccharides were given in different orders according to a Latin-square design. The female volunteer performed the basal and the three study weeks at monthly intervals to control for possible changes in colonic function related to the menstrual cycle (Wald et al. 1981).

The volunteers followed a self-selected diet, restricted in dietary fibre and alcohol content (Table 1), and took the supplements of viscous polysaccharides three times daily in an orange drink. The three polysaccharides tested were guar gum $(15 \mathrm{~g} / \mathrm{d}$; Duche, Lancaster $)$, ispaghula husk ( $14 \mathrm{~g} / \mathrm{d}$; Fybogel, Reckitt \& Colman, Hull) and xanthan gum (15 g/d; Keltrol F grade, Kelco, San Diego, CA).

All stools passed during the basal and test weeks were collected in plastic bags, and labelled with the time and date. These were weighed and the daily faecal mass and stool frequency were calculated. The lower limit for the definition of an increase in faecal mass was $20 \mathrm{~g} / \mathrm{d}$. The lower limit for an increase in stool frequency was 0.14 stools $/ \mathrm{d}$, equivalent to one additional stool per week. The gastrointestinal transit time was measured during these weeks using a continuous method (Cummings et al. 1976) which involved ingesting fifteen radio-opaque markers with breakfast each day, X-raying all stools passed subsequently to measure their excretion, and then fitting this information into a standard equation. The lower limit for a reduction in transit time was taken as $4 \mathrm{~h}$.

A sample of fresh faeces was collected at the end of the basal week and was incubated with the three viscous polysaccharides in vitro, and the results of these incubations were compared with the stool output and transit time.

\section{In vitro incubations of faecal material}

Samples of fresh faeces were homogenized $(50 \mathrm{~g} / \mathrm{l})$ in sterile growth medium and then anaerobically incubated at $37^{\circ}$ for $21 \mathrm{~h}$ with the viscous polysaccharides according to a method previously described (Tomlin et al. 1986). 
(a) Ispaghula

(b) Guar gum

(c) Xanthan gum

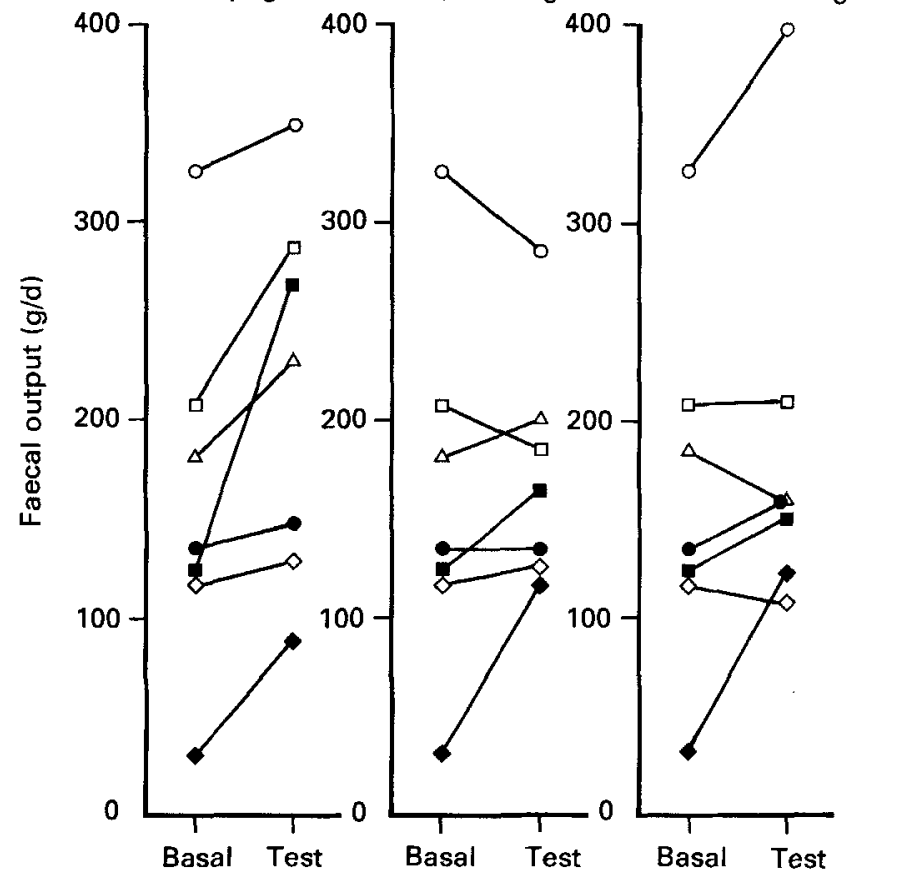

Fig. 1. The changes in faecal output caused by feeding the viscous polysaccharides, $(a)$ ispaghula, $(b)$ guar gum and $(c)$ xanthan gum. For details of dietary regimen, see p. 468 and Table 1. (O), (O), ( $\square$ ), ( $\square)$, $(\triangle),(\diamond),(\diamond)$, Mean values for individual subjects.

The concentrations of polysaccharides in the siurries were $(\mathrm{g} / \mathrm{l})$ guar gum 5 , ispaghula 5 and xanthan gum $2 \cdot 5$. These concentrations were chosen to produce easily measurable viscosities. For each test there were two control cultures; one contained only the faecal homogenate and the other contained only the polysaccharide solution. Samples were analysed for viscosity, using an Ostwald viscometer (Mark, 1945), and $\mathrm{pH}$ (glass electrode, model $10 \mathrm{pH}$ meter; Corning Eel, Medfield, MA, USA) within $20 \mathrm{~min}$ of mixing and after incubation for $21 \mathrm{~h}$. The viscosity was considered to have been removed if after incubation it was equal to or less than the viscosity of water measured on this apparatus ( $2 \cdot 1 \mathrm{mPas})$. Viscosity was considered to have been maintained if the final test value was equal to or higher than the final control value. Between these two extremes the viscosity was considered to have been reduced. The definition of a $\mathrm{pH}$ fall was a reduction by more than the value of the mean rise in $\mathrm{pH}$, which was $0.02 \mathrm{pH}$ units.

Gases produced by fermentation were collected in metallized gas bags (Analysis Automation, Oxford, Oxon). Hydrogen concentration in the gas samples was measured by means of an electrochemical detector (GMI, Renfrew, Scotland; Corbett et al. 1981). Carbon dioxide concentration was measured by gas-liquid chromatography (model F30; Perkin Elmer, Beaconsfield, Bucks) using a column packed with Porapak $Q$ at $32^{\circ}$, a carrier gas of nitrogen $(40 \mathrm{ml} / \mathrm{min})$, an injector temperature of $100^{\circ}$ and a thermal conductivity detector run at $300^{\circ} . \mathrm{H}_{2}$ and $\mathrm{CO}_{2}$ were considered to have been produced if there was more than $15 \mu \mathrm{l}$ or $1 \mathrm{ml}$ respectively above the faeces-alone control value.

\section{Statistical analysis}

Results are expressed as medians, and the statistical significance of differences between sets of measurements was assessed by Wilcoxon's ranked pairs test. Pearson's product-moment 


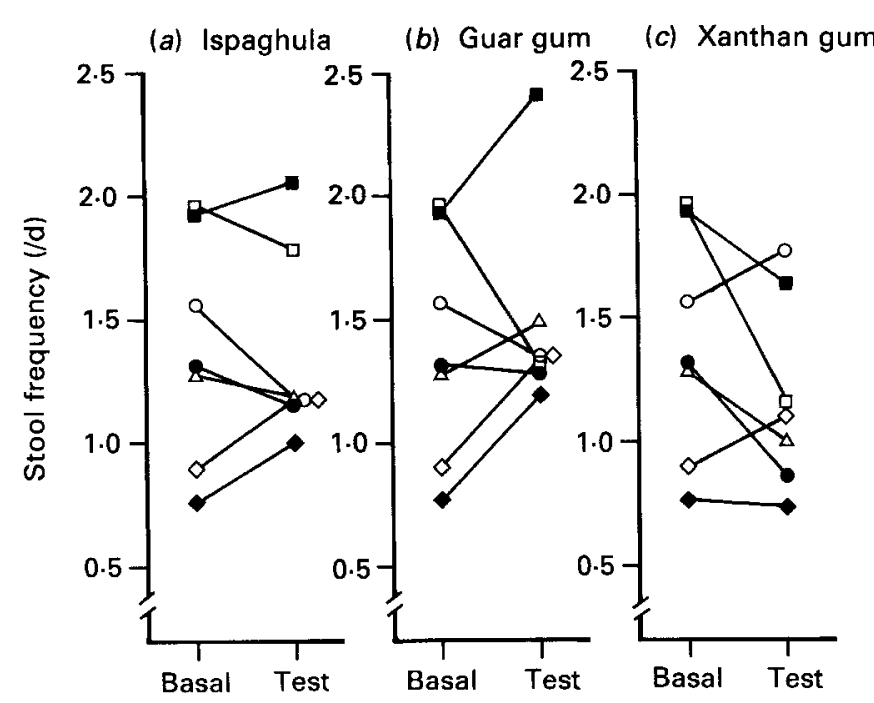

Fig. 2. The changes in stool frequency caused by feeding the viscous polysaccharides, $(a)$ ispaghula, $(b)$

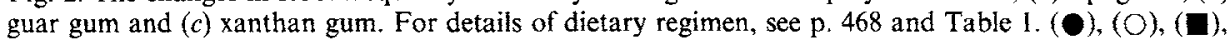
$(\square),(\triangle),(\bullet),(\diamond)$, Mean values for individual subjects.

correlation coefficients (using linear and $\log / \log$ transformation of the values) were calculated to investigate associations between the absolute values, and also the magnitude of the changes in colonic function in vivo. In addition, the changes caused by feeding the three gums to the seven subjects were expressed qualitatively and the $\chi^{2}$ association between various pairs of variables were calculated. The Mann-Whitney $U$ test was used to test differences between groups selected according to these qualitative assessments.

\section{RESULTS}

\section{Stool output, frequency and transit time}

Only ispaghula feeding significantly increased the daily faecal mass $(P<0.05$, Fig. 1$)$. The changes in faecal mass in response to guar gum and xanthan gum showed great intersubject variation. None of the preparations significantly affected the stool frequency or the transit time $(P>0.05$, Figs. 2 and 3$)$.

The absolute values for transit time and faecal mass were inversely related to each other during the basal week $(r-0.90$ for $\log / \log$ relation, $P<0.01)$, during the week of ispaghula feeding $(r-0.85 \log / \log , P<0.02)$, and when all the feeding periods were grouped together $(r-0.73 \log / \log , P<0.001)$, but not during the weeks of giving guar gum and xanthan gum. The changes in faecal mass observed during feeding with any of the polysaccharides were not significantly correlated with the corresponding changes in transit time $(r-0 \cdot 12, P>0.05)$; increases in stool mass observed on feeding the polysaccharides were associated with decreases in transit time on only six of eleven occasions.

Stool frequency was significantly correlated with transit time $(r-0.67, P<0.05)$ when all feeding periods were grouped together, but not with faecal mass $(r 0.42, P>0.05)$. Similarly the change in stool frequency on feeding a polysaccharide was inversely related to the change in transit time $(r-0.58, P<0.01)$ but was not related to a change in faecal mass $(r 0.38, P>0.05)$. 
(a) Ispaghula

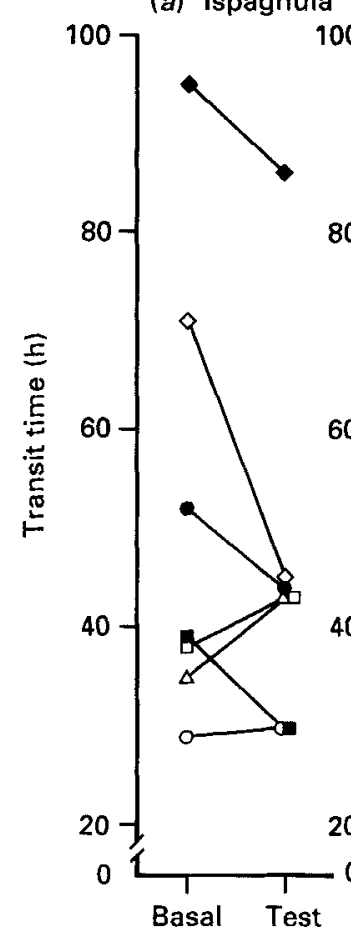

(b) Guar gum

(c) Xanthan gum
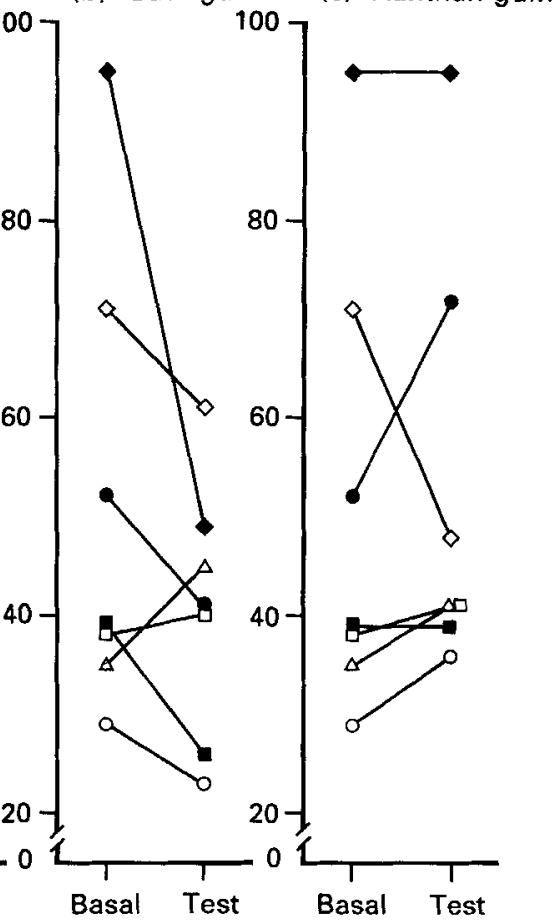

Fig. 3. The changes in transit time caused by feeding the viscous polysaccharides, $(a)$ ispaghula, $(b)$ guar gum and $(c)$ xanthan gum. For details of dietary regimen, see p. 468 and Table $1 .(O),(0),(\square),(\square)$, $(\triangle),(\diamond),(\diamond)$, Mean values for individual subjects.

\section{In vitro incubations}

Guar gum was rapidly fermented in vitro by the faeces from all seven volunteers, with concomitant removal of viscosity, reduction in $\mathrm{pH}$ and generation of gases (Fig. $4(a)$ ). Ispaghula was fermented, resulting in a reduction in $\mathrm{pH}$ in all cultures and gas production from most, but the viscosities were not completely removed, although they were lower than the final control value in five of seven cases (Fig. 4(b)). The results of xanthan gum incubations showed considerable individual variations, ranging from complete fermentation (one person) to no evidence of fermentation from any of the variables studied (four people) (Fig. 4(c)).

\section{Associations between in vitro incubations and in vivo values}

Slurries in which the viscosity was reduced or removed during in vitro incubation were associated with a significantly faster transit time on feeding the gum than slurries where the viscosity was maintained (Mann-Whitney $U$ test, $P<0.05$ ). These two factors were also significantly associated in the $\chi^{2}$ test $(P<0.05)$. In fact, feeding a gum to subjects whose faecal bacteria were unable to reduce the viscosity of that gum never resulted in a reduction in transit time (Fig. 5).

The relation between the effect on viscosity in vitro and the change in faecal mass was slightly different; greater increases in faecal mass occurred in association with those cultures in which the viscosity was either unaffected or reduced but not totally removed than with cultures in which the viscosity was totally removed $(P<0.05)$. 

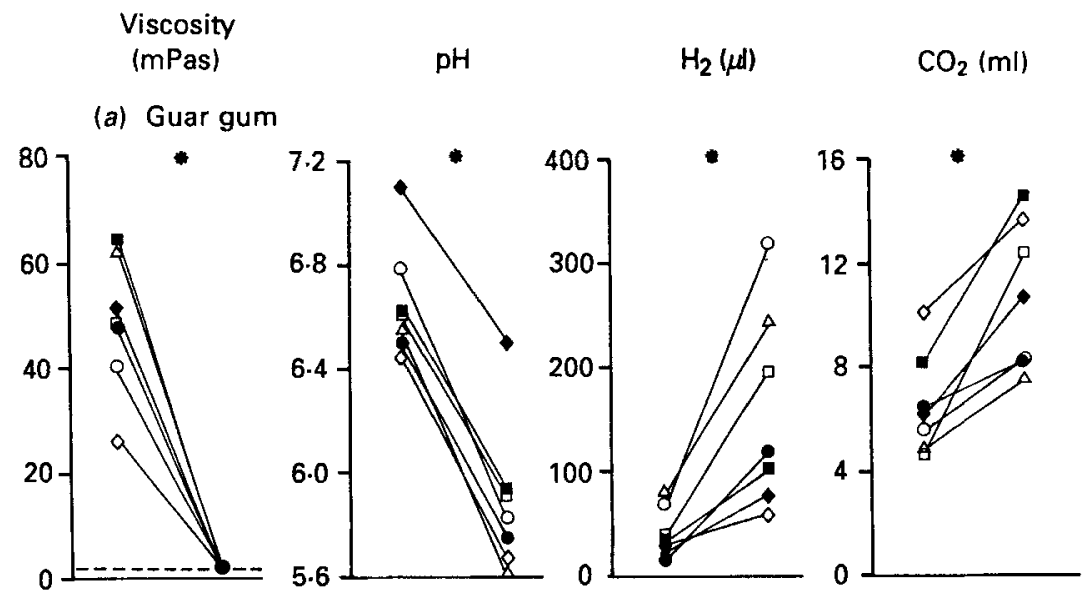

(b) Ispaghula
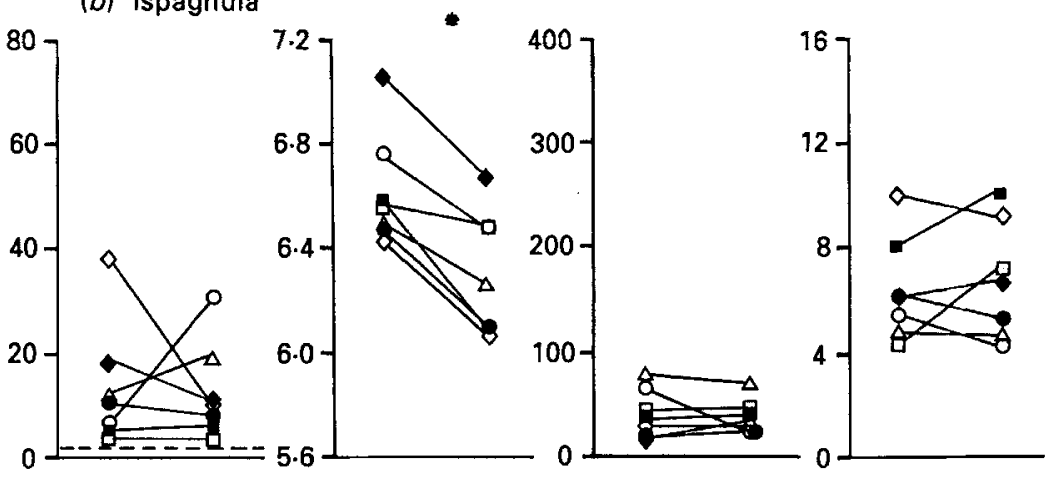

(c) Xanthan gum
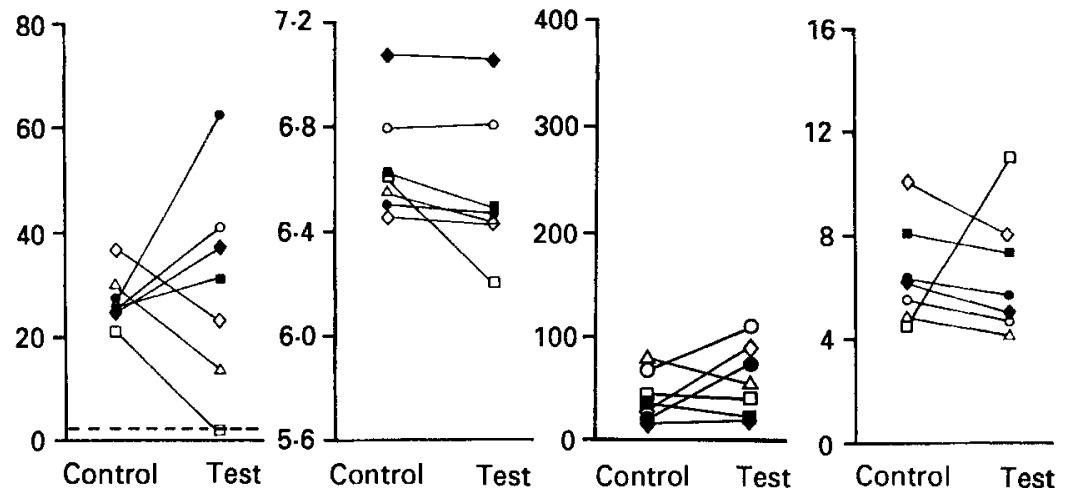

Fig. 4. The final values of viscosity (mPas) and $\mathrm{pH}$, and the volumes of hydrogen $(\mu \mathrm{l})$ and carbon dioxide $(\mathrm{ml})$ produced from control and test cultures from each subject during incubation with $(a)$ guar gum, $(b)$ ispaghula and $(c)$ xanthan gum. ${ }^{*}$ Mean values significantly different from control value $(P<0.05)$. (--) represents $2 \cdot 1 \mathrm{mPas}$, the viscosity of water measured with this apparatus. For details of procedures see p. $469 .(\bigcirc),(\bigcirc),(\square),(\square),(\Delta),(\diamond),(\diamond)$, Mean values for individual subjects. 


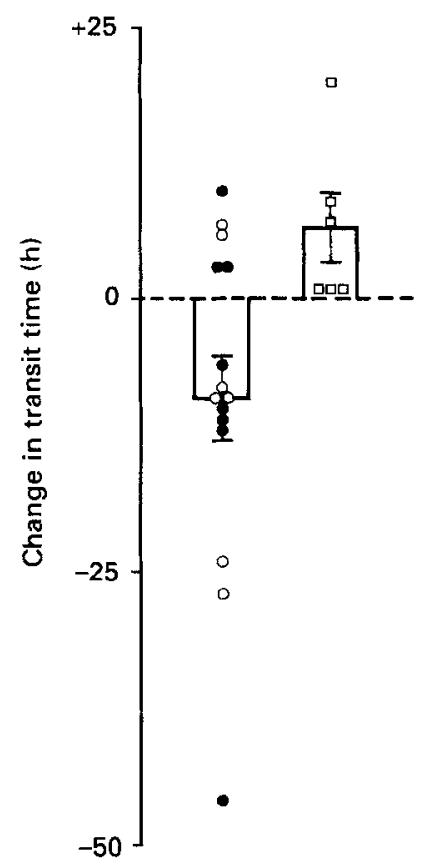

Fig. 5. Values for the change in transit time of different groups (means with their standard errors represented by vertical bars) sorted qualtitatively according to in vitro effects on the viscosity; ( $\square$ ), viscosity was removed; $(O)$, viscosity reduced; $(O)$, viscosity maintained $(P<0.05$, Mann-Whitney $U$ test).

The effect of giving a polysaccharide on the stool frequency was related to the release of $\mathrm{H}_{2}$ gas from the cultures; $\mathrm{H}_{2}$ production was associated with a higher stool frequency than if no $\mathrm{H}_{2}$ was produced $(P<0.05)$.

Fermentation, defined as a decrease in viscosity plus a decrease in $\mathrm{pH}$ and the production of gases, was associated with a reduction in transit time $\left(\chi^{2}, P<0.05\right)$, but not with changes in faecal mass or stool frequency.

\section{DISCUSSION}

The commonly reported inverse relation between transit time and faecal mass (Burkitt et al. 1972; Stasse-Wolthius et al. 1979) has been explained by differences in the intake of dietary fibre, as a high-fibre diet or administration of wheat bran increases faecal mass and accelerates transit (Burkitt et al. 1972), and people with a low intake of fibre tend to have slow colonic transit and low faecal output.

Although our results confirmed the relation between transit time and faecal mass under basal conditions, we found that feeding viscous polysaccharides could affect one of these factors without necessarily influencing the other, suggesting that transit time and faecal mass are not necessarily dependent variables. Moreover, stool frequency was significantly associated with transit time and not with faecal mass.

There was great individual variability among the responses of each of the measurements to feeding each of the gums. Of the three polysaccharides tested only ispaghula increased stool mass, and the inverse relation between stool mass and transit time was maintained after ispaghula feeding, but not with the other polysaccharides. In vitro fermentations were 
carried out in an attempt to determine whether the individual's capacity to ferment a particular polysaccharide was related to the effect that feeding that polysaccharide had on faecal mass and transit time. The findings from all feeding periods were then pooled so that $\chi^{2}$ tests could be used to assess the predictive power of in vitro fermentations to determine the effect of a polysaccharide on stool output. Our results showed that a greater increase in faecal mass occurred when some polysaccharide structure was preserved, as indicated by incomplete removal of viscosity. This suggests that the major mechanism by which the soluble polysaccharides tested increased faecal mass is through holding water rather than the stimulation of bacterial growth.

Decreases in transit time, however, appear to depend on some bacterial degradation (causing either partial or total loss of viscosity), and were associated with fermentation in the appropriate cultures. 'Thus it is possible that the end-products of fermentation stimulate propulsive colonic motility. Gases produced may increase the volume of colonic contents and so promote propulsion; the arrival of gas in a rectum containing faeces may signal a desire to defaecate by stimulating tension receptors, accounting for the observed relation between stool frequency and in vitro $\mathrm{H}_{2}$ production. In vitro experiments using preparations of rat colon and in vivo measurements in sheep suggest that short-chain fatty acids can stimulate contractions of colonic smooth muscle at concentrations as low as $1.0 \mathrm{~mm}$ (Svendsen, 1972; Yokokura et al. 1977), but it is not certain whether such contractions are propulsive. At higher concentrations of $10-200 \mathrm{~mm}$, which are closer to the order of magnitude of levels normally found in the colon, short-chain fatty acids seem to stimulate mixing activity and inhibit propulsive events in both the sheep and the horse in vivo (Svendsen, 1972; Candau \& Vigroux, 1974; Gregory, 1984). This may explain why there were no significant associations between in vitro $\mathrm{pH}$ (which partially reflects shortchain fatty acid production) and the transit time, stool frequency or mass.

Of the three substances tested, only ispaghula is established as a bulk laxative; neither of the other two fibres tested have, at acceptable doses, been shown to increase consistently the production of faeces. The effectiveness of ispaghula as a bulk laxative may be explained by its fermentation characteristics; the retention of some of its structure accounts for its effect on stool output, while the fact that it is partially fermented would cause, in addition, an acceleration of transit time. Wheat bran is another type of fibre that is only partially degraded by colonic bacteria; it increases stool output, accelerates transit and is regarded as an effective bulk laxative.

In summary, the findings suggest that viscous polysaccharides can influence stool mass and transit time independently, and these effects depend on the extent to which they are degraded by the colonic bacteria. Increases in faecal output are associated with some retention of polysaccharide structure, while acceleration of transit time depends on some bacterial degradation. The most effective bulking agents are probably partially fermented (accelerating transit time) but retain enough of their structure to increase faecal mass.

\section{REFERENCES}

Burkitt, D. P., Walker, A. R. \& Painter, N. S. (1972). Lancet ii, 1408-1411.

Candau, M. \& Vigroux, P. (1974). Comptes Rendus Societe de Biologie 168, 893-897.

Corbett, C. L., Thomas, S., Read, N. W., Hobson, N., Bergman, I. \& Holdsworth, C. D. (1981). Gut 22, 836-840.

Cummings, J. H., Jenkins, D. J. \& Wiggins, H. S. (1976). Gut 17, 210-218.

Eastwood, M. A., Brydon, W. G. \& Anderson, D. M. W. (1986). American Journal of Clinical Nutrition 44, 5I-55.

Gregory, P. L. (1984). Journal of Physiology 346, 379-393.

Mark, H. (1945). In Physical Methods of Organic Chemistry, vol 1, pp. 135-147 [A. Weissburger, editor]. New York: Intersciences. 
Prynne, C. J. \& Southgate, D. A. T. (1979). British Journal of Nutrition 41, 495-503.

Stasse-Wolthius, M., Hautvast, J. G., Hermus, R. J., Katan, M. B., Bausch, J. E., Reitberg-Brussard, J. H., Velema, J. P., Zondervan, J. H., Eastwood, M. A. \& Brydon, W. G. (1979). American Journal of Clinical Nutrition 32, 1881-1888.

Svendsen, P. (1972). Nordisk Veterinaer Medicin 24, 393-396.

Tomlin, J. (1987). Scandinavian Journal of Gastroenterology 22, Suppl. 129, 100-104.

Tomlin, J., Read, N. W., Edwards, C. A. \& Duerden, B. I. (1986). British Journal of Nutrition 55, 481486.

Wald, A., Van Thiel, D. H., Hoechstetter, L., Gavaler, J. S., Egler, K. M., Verm, R., Scott, L. R. \& Lester, R. (1981). Gastroenterology 80, 1497-1500.

Yokokura, T., Yajima, T. \& Hashimoto, S. (1977). Life Sciences 21, $59-62$. 